\title{
Tertiary Cancer Center Experience: Pregnancy-Associated Breast Cancer
}

\author{
Taha Y. KUZAN ${ }^{1}$, Emre KOCA ${ }^{1}$, Taner BABACAN ${ }^{1}$, Ibrahim H. TURKBEYLER ${ }^{2}$, \\ Furkan SARICI ${ }^{1}$, Kadri ALTUNDAG ${ }^{1}$ \\ ${ }^{1}$ Hacettepe University Cancer Institute, Department of Medical Oncology, Ankara \\ ${ }^{2}$ Adıyaman University Faculty of Medicine, Department of Internal Medicine, Adıyaman, TURKEY
}

To the Editor,

Breast carcinoma that is diagnosed during pregnancy or within 1 year postpartum classified as pregnancyassociated breast cancer and it is the second most common malignancy associated with pregnancy accounting approximately 1 in 3,000 to 10,000 pregnancies. ${ }^{1}$ Breast cancer during pregnancy is a very important clinical antitiy for not only the patient but also for unborn child especially when regarding treatment modalities. In literature, knowledge about pregnancyassociated breast cancer is limited to rectospective studies and case reports.

Here we are reporting clinicopathological charasteristics and treatment outcomes of pregnancy-associted breast cancer patients. We have looked rectospective- ly 2578 breast cancer patients who diagnosed in Hacettepe University Hospital between 2003 and 2012. Eight $(0.3 \%)$ of those patients had pregnancy associated breast cancer.

The mean age of patients was 36 (30-43). Seven patients had invasive ductal carsinoma and one patient had tubular carsinoma. 6 of 8 patients had T2/T3 stage. Totally 6 patients were grade III and 3 patients were having metastatic tumors when they were first diagnosed. 5 of 8 patients were both positive for estrogen and progesterone receptors. 2 patient were cerbB 2 positive (Table 1). 6 of 8 patients were undergone modified radical mastectomy and all of 8 patients had chemotherapy.

\begin{tabular}{|c|c|c|c|c|c|c|c|c|c|}
\hline & Age & Diagnose time & Histology & Grade & ER/PR & HER2 & pT & $\mathbf{N}$ & M \\
\hline Patient 1 & 44 & Pospartum 5.mo & IDC & III & $-/-$ & + & 2 & N3-11\15 & 0 \\
\hline Patient 2 & 29 & Pregnancy 4.mo & IDC & III & $+/+$ & - & $x$ & NX & 1 \\
\hline Patient 3 & 36 & Pregnancy 6.mo & IDC & III & $-/-$ & - & 3 & N3-28\32 & 0 \\
\hline Patient 4 & 34 & Pregnancy 6.mo & Tubular & I & $+/+$ & - & 1 & No-0\32 & 0 \\
\hline Patient 5 & 40 & Pregnancy 7.mo & IDC & III & $+/+$ & + & 2 & No-0126 & 0 \\
\hline Patient 6 & 35 & Postpartum12.mo & IDC & $\|$ & $+/+$ & - & 2 & NX-PN1-1\42 & 1 \\
\hline Patient 7 & 34 & Pregnancy 7.mo & $\begin{array}{l}\text { IDC-NON- } \\
\text { BAZALOID }\end{array}$ & III & $-/-$ & - & 3 & No-0\19 & 1 \\
\hline Patient 8 & 38 & Pregnancy 1.mo & IDC & III & $+/+$ & - & 2 & CN2-pn0-0\8 & 0 \\
\hline
\end{tabular}


International Journal of Hematology and Oncology

\begin{tabular}{|c|c|c|c|c|c|c|c|}
\hline & Chemotherapy & CT-Protocol & Hormone & $\begin{array}{l}\text { Radio } \\
\text { therapy }\end{array}$ & $\begin{array}{l}\text { Surgery } \\
\text { therapy }\end{array}$ & Life status & Follow up \\
\hline Patient 1 & Adjuvant & $\begin{array}{l}\text { CAF X4 } \\
\text { Docetaxel X4 } \\
\text { Herceptin x9 }\end{array}$ & None & Yes & MRM & Alive & 72 \\
\hline Patient 2 & Metastatic & $\begin{array}{l}\text { TAC X6 } \\
\text { Lucrine X3 }\end{array}$ & Tamoxifen X5 & Yes & & Alive & 38 \\
\hline Patient 3 & Adjuvant & TAC X6 & None & Yes & MRM & Alive & 35 \\
\hline Patient 4 & Adjuvant & CMF X6 & Tamoxifen & Yes & MRM & Alive & 31 \\
\hline Patient 5 & Adjuvant & $\begin{array}{l}\text { Docetaxel X4 } \\
\text { Herceptin X4 } \\
\text { Docetaxel X4 } \\
\text { Cphosphamid X4 } \\
\text { Herceptin } 1 \text { year }\end{array}$ & $\begin{array}{l}\text { Tamoxifen } 9 \text { mo } \\
\text { Lucrine } 9 \text { mo }\end{array}$ & Yes & & Alive & 28 \\
\hline Patient 6 & Metastatic & $\begin{array}{l}\text { TAC X6 } \\
\text { Lucrine X2 }\end{array}$ & Tamoxifen X5 & Yes & MRM & Alive & 29 \\
\hline Patient 7 & Metastatic & $A C \times 4$ & None & Yes & MRM & Alive & 18 \\
\hline Patient 8 & Neoadjuvant & $\begin{array}{l}\text { TAC X6 } \\
\text { Capasitabine X6 }\end{array}$ & Tamoxifen X5 & Yes & MRM & Alive & 15 \\
\hline
\end{tabular}

One patient had neoadjuvant therapy, Four patient had TAC(6), 1 patient had AC(4), 1 patient had $\mathrm{CMF}(6), 1$ patient had $\mathrm{CAF}(4)$, Docetaxel(4), 1 patient had taxoter protocol as treatment. All of patients had either antracycline or taxane based chemotherapy but only half of them had hormone receptor therapy (Table 2). Mean follow up was 33 months (15-72) and all patients were alive.

Pregnancy associated breast cancers makes only $\% 5$ of all breast cancer patients younger than 50 years of age. However they account for approximetly \% 20 of all breast cancers seen before age $30 .^{1,2}$ The prevalance of breast cancer in pregnancy is still low but it is increasing due to delay in childbearing and wider use of screening. Tumor charasteristics of breast cancers in pregnancy are generally poor and they are usually have advanced stage..$^{3-5}$ In our study most of the patients were grade III and 3 of them were metatstatic at the time of diagnosis. Physicians must be aware of breast cancer when they encounter with a breast mass in a pregnant patient. In clinically suspicious cases, biopsy should be in mind as a diagnostic tool even if mammograpy or ultrasound is negative. Pregnant patients with breast cancer must be handled multidisciplinary with an oncologist and obstetrician.

\section{REFERENCES}

1. Antonelli NM, Dotters DJ, Katz VL, et al. Cancer in pregnancy: a review of the literature. Part I. Obstet Gynecol Surv 51: 125134, 1996.

2. Wallack MK, Wolf JA Jr, Bedwinek J, et al. Gestational carcinoma of the female breast. Curr Probl Cancer 7: 1-58, 1983.

3. Anderson BO, Petrek JA, Byrd DR, et al. Pregnancy influences breast cancer stage at diagnosis in women 30 years of age and younger. Ann Surg Oncol 3: 204-211, 1996.

4. Middleton LP, Amin M, Gwyn K, et al. Breast carcinoma in pregnant women: assessment of clinicopathologic and immunohistochemical features. Cancer 98: 1055-1060, 2003.

5. Reed W, Hannisdal E, Skovlund E, et al. Pregnancy and breast cancer: a population-based study. Virchows Arch 443: 44-50, 2003.

\section{Correspondence}

Dr. Ibrahim Halil TÜRKBEYLER

Adiyaman Üniversitesi Tip Fakültesi

Iç Hastaliklari Anabilim Dali

02000, ADIYAMAN / TURKEY

Tel: (+90.416) 2233815

e-mail: turkbeyler@mynet.com 